\title{
Tailoring glucocorticoids in patients with severe COVID-19: a narrative review
}

\author{
Ming-Hao Luo ${ }^{1 \#}$, Yi-Qi Qian ${ }^{2 \#}$, Dan-Lei Huang ${ }^{1 \#}$, Jing-Chao Luo ${ }^{2}$, Ying Su ${ }^{2}$, Huan Wang ${ }^{2}$, Shen-Ji Yu ${ }^{2}$, \\ Kai Liu ${ }^{2}$, Guo-Wei Tu ${ }^{2}$ Zhe Luo, \\ ${ }^{1}$ Shanghai Medical College, Fudan University, Shanghai, China; ${ }^{2}$ Department of Critical Care Medicine, Zhongshan Hospital, Fudan University, \\ Shanghai, China; ${ }^{3}$ Department of Critical Care Medicine, Xiamen Branch, Zhongshan Hospital, Fudan University, Xiamen, China \\ Contributions: (I) Conception and design: MH Luo, YQ Qian, GW Tu, Z Luo; (II) Administrative support: GW Tu, Z Luo; (III) Provision of study \\ materials or patients: None; (IV) Collection and assembly of data: JC Luo, Y Su, H Wang, SJ Yu; (V) Data analysis and interpretation: DL Huang; (VI) \\ Manuscript writing: All authors; (VII) Final approval of manuscript: All authors. \\ \#These authors contributed equally to this work. \\ Correspondence to: Zhe Luo; Guo-Wei Tu. Department of Critical Care Medicine, Zhongshan Hospital, Fudan University, Shanghai 200032, China. \\ Email: luo.zhe@zs-hospital.sh.cn; tu.guowei@zs-hospital.sh.cn.
}

Objective: To discuss the pathogenesis of severe coronavirus disease 2019 (COVID-19) infection and the pharmacological effects of glucocorticoids (GCs) toward this infection. To review randomized controlled trials (RCTs) using GCs to treat patients with severe COVID-19, and investigate whether GC timing, dosage, or duration affect clinical outcomes. Finally. to discuss the use of biological markers, respiratory parameters, and radiological evidence to select patients for improved GC therapeutic precision.

Background: COVID-19 has become an unprecedented global challenge. As GCs have been used as key immunomodulators to treat inflammation-related diseases, they may play key roles in limiting disease progression by modulating immune responses, cytokine production, and endothelial function in patients with severe COVID-19, who often experience excessive cytokine production and endothelial and reninangiotensin system (RAS) dysfunction. Current clinical trials have partially proven this efficacy, but GC timing, dosage, and duration vary greatly, with no unifying consensus, thereby creating confusion.

Methods: Publications through March 2021 were retrieved from the Web of Science and PubMed. Results from cited references in published articles were also included.

Conclusions: GCs play key roles in treating severe COVID-19 infections. Pharmacologically, GCs could modulate immune cells, reduce cytokine and chemokine, and improve endothelial functions in patients with severe COVID-19. Benefits of GCs have been observed in multiple clinical trials, but the timing, dosage and duration vary across studies. Tapering as an option is not widely accepted. However, early initiation of treatment, a tailored dosage with appropriate tapering may be of particular importance, but evidence is inconclusive and more investigations are needed. Biological markers, respiratory parameters, and radiological evidence could also help select patients for specific tailored treatments.

Keywords: Coronavirus disease 2019 (COVID-19); glucocorticoid (GC); cytokines; C-reactive protein (CRP); computed tomography

Submitted Apr 11, 2021. Accepted for publication Jun 10, 2021.

doi: 10.21037/atm-21-1783

View this article at: https://dx.doi.org/10.21037/atm-21-1783

$\wedge$ ORCID: 0000-0002-3109-7883. 


\section{Introduction}

Coronavirus disease 2019 (COVID-19) has become an unprecedented worldwide challenge. To date, over 130 million people have been infected and almost three million have died (1). As cases surge, intensive care units (ICUs) in many countries are facing huge pressures to treat critically ill patients, concomitant with on-going governmental efforts to tackle the pandemic.

The first report from the RECOVERY group in July 2020 provided a potentially promising glucocorticoid (GC) treatment regimen for patients infected with COVID-19 (2). Dexamethasone resulted in a lower 28-day mortality among those receiving either invasive mechanical ventilation (IMV) or oxygen alone, suggesting GCs may be effective in some populations (2).

While controversy continues over whether GCs may be a definitive treatment, updated World Health Organization (WHO) guidelines now recommend using them in clinical practice (3). However, evidence gaps exist as there have been no prospective trials specifically reporting on GC timing, dosage, or duration. Thus, uncertainties around GCs must be clarified for prescribing physicians and patients.

In this review, we discuss the pathogenesis of severe COVID-19 infection and the pharmacological effects of GCs toward this infection. We review randomized controlled trials (RCTs) using GCs to treat patients with severe COVID-19, and investigate whether GC timing, dosage, or duration affect clinical outcomes. Finally, we discuss the use of biological markers, respiratory parameters, and radiological evidence to select patients for improved GC therapeutic precision.

We present the following article in accordance with the Narrative Review reporting checklist (available at https:// dx.doi.org/10.21037/atm-21-1783).

\section{Methods}

PubMed and Web of Science were searched using the terms "glucocorticoid", "corticosteriod", "steriod" and "COVID-19" in English through March 2021. Articles in English and in Chinese were included. We also included results from cited references in published articles searched by using the method described above. The final reference list was generated on the basis of relevance and originality with regard to the topics covered in this review.

\section{The pathogenesis of severe COVID-19}

Severe acute respiratory syndrome coronavirus 2 (SARSCoV-2), the pathogen causing COVID-19, initially binds to host cells expressing the surface receptor, angiotensinconverting enzyme 2 (ACE2) (4). Multiple subsequent reactions are involved in pathogenesis, producing a unique pathophysiological landscape contributing to COVID-19 infection (Figure 1).

\section{Cytokine production}

Cytokine production is an important element of immune response initiated in infected individuals. Upon binding to SARS-CoV-2, cells expressing ACE2, including alveolar epithelial cells, undergo pyroptosis and release damage associated molecular patterns (4) consistent with postmortem histopathological findings characterized by diffuse alveolar damage (DAD) (5-7). This damage is recognized by monocytes and macrophages which trigger proinflammatory cytokine and chemokine cascades (4).

Elevated cytokine levels, particularly in severely ill patients, suggest that excessive cytokine production may contribute to COVID-19 pathogenesis $(8,9)$. Increased interleukin (IL) levels, including IL-2, IL-6, IL-7, interferon- $\gamma$ (IFN- $\gamma$ ), tumor necrosis factor- $\alpha(\mathrm{TNF}-\alpha)$, and monocyte chemoattractant protein-1 (MCP-1) are observed during COVID-19 infection (10,11). The term "cytokine storm" has been used to describe this distinctive immune response (12). Among these cytokines, increased IL-6 levels are highly associated with shorter patient survival times (13). These observations suggest that balancing immune responses after infection are vital to successful treatment outcomes.

\section{Endothelial dysfunction}

As ACE2 receptors are primarily located on endothelial cells in the lungs, they are the main victims in SARS-CoV-2 infection. Numerous circulating markers of endothelial injury, such as coagulation factor VIII, von-Willebrandfactor, and angiopoietin 2 are increased in patients with COVID-19 (14). Similarly, autopsies have also revealed the formation of hyaline membranes and micro-thromboses as key pathological patterns besides DAD $(5,7)$.

Endotheliopathy reflects micro-thrombosis in end- 


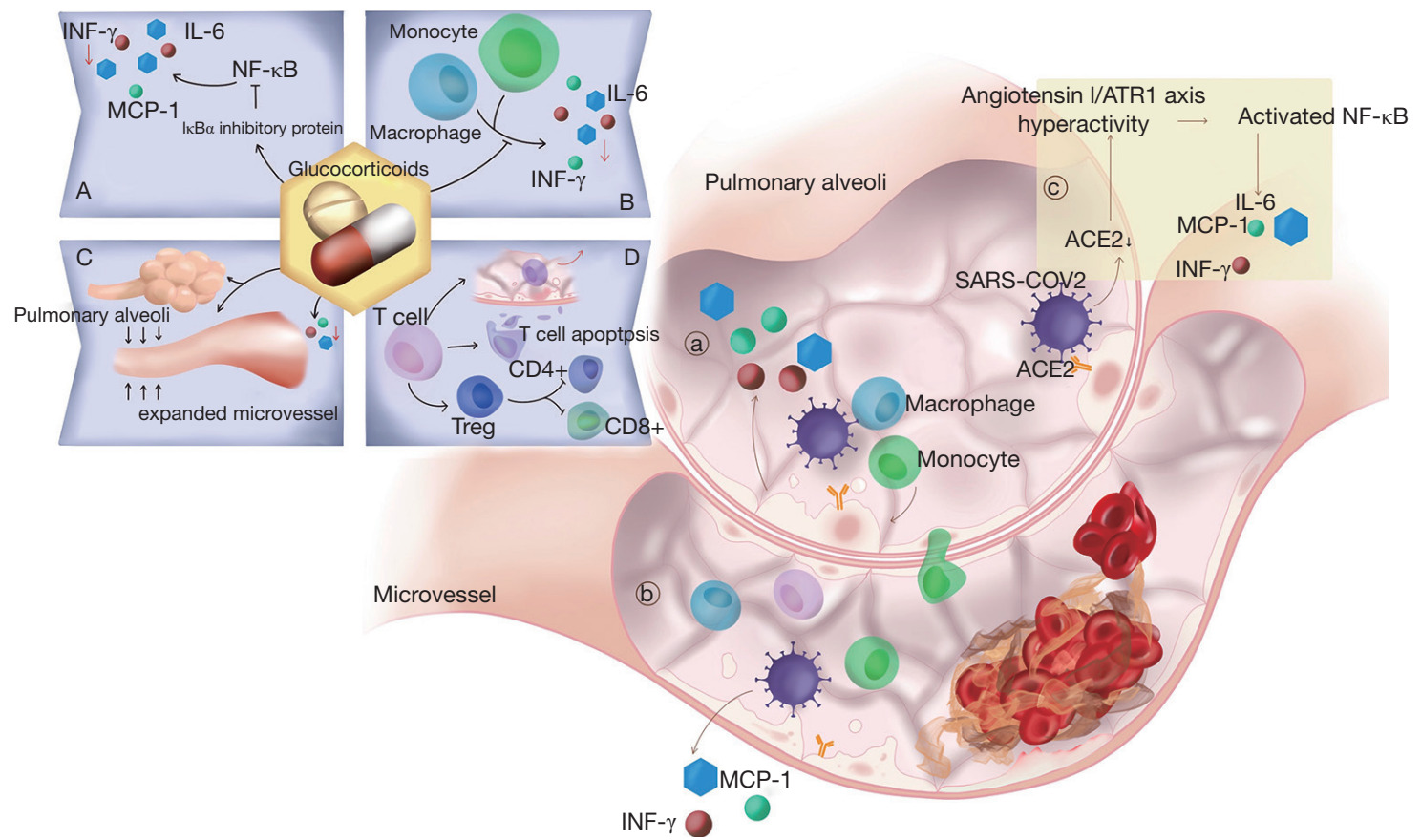

Figure 1 The pathogenesis of severe COVID-19 and the pharmacology of glucocorticoid (GC). Multiple reactions may be involved in the pathogenesis of COVID-19 and tend to coordinate with each other. GC can target these reactions pharmacologically. (a) Alveolar epithelial cells infected by SARS-CoV-2 undergo pyroptosis and release damage associated molecular patterns which are recognized by neighbouring monocytes and macrophages, triggering the generation of pro-inflammatory cytokines and chemokines. (b) Infected vascular endothelial cells threaten the integrity of vessels, leading to infiltration of leukocytes to lung tissue. Besides, numerous markers are released, facilitating the formation of thrombosis. Cytokines are also released because of damaged vascular endothelial cells. (c) ACE2 downregulation leads to a hyperactivity of the angiotensin II/ATR1 axis. resulting in the binding of angiotensin II to ATR1. Nuclear factor kappa B (NF- $\mathrm{kB}$ ) is then

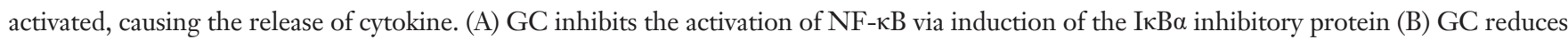
cytokine production among macrophages and monocytes by the genomic effect (C) GC restricts the extent of endotheliopathy through cytokine reduction, barrier enhancement and vascular modification. (D) GC exerts unique actions on T cells. First, GC decreases the number of circulating $\mathrm{T}$ cells by favoring their migration back to the bone marrow and secondary lymphoid tissues. It also induces apoptosis of $\mathrm{T}$ cells in peripheral lymphoid organs and down-regulates adhesion molecule. In addition, GCs increases the frequency of regulatory T cells (Treg), which inserts an immunosuppressive effect on the activation, proliferation and cytokine production of CD4+ T cells and CD8+ T cells.

organ damage, and mediates inflammatory cell infiltration particularly in T cells in the lungs (15). Complex interactions between coagulopathy, thrombocytopathy, and endotheliopathy contribute to COVID-19-associated thrombo-inflammation $(16,17)$. Also, diffuse infiltration of alveolar walls by CD4+ and CD8+ T lymphocytes has been identified in patient autopsies $(7,18)$. Local inflammation aggravation involving increased pro-inflammatory cytokine and chemokine secretion into patient blood attracts immune cells, notably $\mathrm{T}$ lymphocytes from the blood to infected sites, generating lymphopenia in the peripheral blood $(4,11,19)$. Such coordinated activation of inflammatory and thrombotic responses is a major cause of morbidity and mortality (16). Thus, early identification of endotheliopathy and strategies to mitigate its progression may improve COVID-19 outcomes (20).

\section{Renin-angiotensin system (RAS) dysfunction}

SARS-CoV-2 enters respiratory epithelial cells via ACE2 interactions, causing receptor internalization and subsequent down-regulation (21). Reduced ACE2 levels, which have been shown to regulate RAS, could lead to RAS dysfunction, potentially enhancing inflammation and airway vascular permeability (22). ACE2 down-regulation reduces the transformation of angiotensin II to angiotensin-(1-7), 
thus causing hyperactivity of the angiotensin II/angiotensin receptor 1 (ATR1) axis (23), resulting in angiotensin II binding to ATR1. This reaction activates nuclear factor- $\mathrm{\kappa B}$ (NF- $\mathrm{kB})$ (24), which is the most important checkpoint in COVID-19-related pro-inflammatory events, with potential roles in COVID-19-related cytokine storms $(25,26)$. Activated NF- $\kappa B$ leads to the downstream production of several pro-inflammatory cytokines, including IL-1, IL-6, and TNF- $\alpha(25,27,28)$, which potentially enhance inflammation and airway vascular permeability (22).

\section{The pharmacological effects of GCs on severe COVID-19}

Several signaling pathways are impacted by GCs that affect cellular activities, with most reactions mediated by glucocorticoid receptors (GRs) (29). In the absence of GCs, GRs reside in the cytoplasm and are complexed with chaperone molecules composed of heat shock proteins (HSP) 90, 70, and immunophilins (30). The GR $\alpha$ is of particular importance in critical illnesses thanks to its dominant effect on GC-mediated activities (31).

GCs enter cells as free molecules and produce biological effects via three pathways (genome, non-genome, and mitochondrial). In the classic genomic pathway, upon cytoplasmic entry, GCs bind to GRs, which induce conformational changes permitting GRs to dissociate from chaperone molecules and dimerize (32). These dimerized complexes are then actively transported to the nucleus where they bind to palindromic GC response elements (GRE) in gene promoters, permitting the subsequent recruitment of co-activators leading to chromatin remodeling and downstream transcription $(32,33)$.

\section{Immune cell modulation}

Cytokine production inhibition by macrophages and monocytes is a major GC anti-inflammatory mechanism (34). Macrophages and monocytes are among the most effective producers of pro-inflammatory mediators. Through genomic effects, GCs inhibit IL-1 $\beta$, IL-6, IL-12, and TNF $\alpha$ transcription and down-regulate chemokine expression, including IL-8 and MCP-1 (34) to limit overwhelming and sustained inflammation.

GCs also exert specific immunosuppressive actions on T cells (35). Circulating T cell numbers are decreased by GCs which promote their migration back to bone marrow and secondary lymphoid tissue. GCs also induce $\mathrm{T}$ cell apoptosis in peripheral lymphoid organs and down-regulate adhesion molecules (36-38). These effects potentially reduce $\mathrm{T}$ cell infiltration to inflamed areas, especially in the lungs of patients with COVID-19. Also, GC treatment increases regulatory $\mathrm{T}$ cell (Treg) frequency (39), in part by enhancing Treg cell numbers or activity, and promoting the development of IL10-producing $\mathrm{T}$ cells (40). This exerts immunosuppressive effects on the activation, proliferation, and cytokine production of CD4+ T cells and CD8+ T cells (41).

\section{Cytokine reduction and inbibition}

GCs modulate cytokine expression via a combination of genomic mechanisms. The activated GR complex binds to and inactivates key pro-inflammatory transcription factors, such as AP-1, whereas it up-regulates the expression of cytokine inhibitory proteins via its GRE, and reduces the half-life and utility of cytokine mRNAs $(42,43)$. Additionally, GCs are potent NF- $\kappa \mathrm{B}$ activation inhibitors (44). This process is important in RAS dysfunction, and is mediated by induction of the inhibitor of nuclear factor- $\mathrm{\kappa B}$ $(\mathrm{I} \kappa \mathrm{B} \alpha)$ protein trapping activated $\mathrm{NF}-\kappa \mathrm{B}$ in inactive cytoplasmic complexes (45).

\section{Improvements in endothelial function}

Inflammatory cell infiltration and increases in vascular permeability and diameter are important characteristics in endotheliopathy (46), whereas GCs restrict the extent of endotheliopathy via cytokine reduction, barrier enhancement, and vascular modification.

As GCs modulate immune cellular activities and act on specific pathways to reduce inflammatory mediators, they inhibit the attraction and infiltration of immune cells to specific inflammation tissue sites by reducing cytokine and chemokine release (47). GC enhancement of barrier function at the lung endothelium is also required to suppress vascular leakage and infiltration of inflammatory cells into the lung $(48,49)$. GCs preserve endothelial barrier integrity by upregulating junctional proteins such as occludin (50) and down-regulating matrix metallopeptidase 9 (MMP-9) which is an enzyme involved in junctional protein cleavage (51-53). GCs also indirectly modify vascular diameter by inhibiting inflammatory mediators to reduce vessel swelling (54).

\section{GCs and patients with severe COVID-19}

GCs have long been considered potential immunomodulators 
Table 1 RCTs regarding severe COVID-19 patients treated with GCs

\begin{tabular}{|c|c|c|c|c|c|c|}
\hline Author & $\begin{array}{l}\text { Size } \\
\text { T/C }\end{array}$ & $\begin{array}{c}\text { Type of } \\
\text { GC }\end{array}$ & $\begin{array}{l}\text { Timing median } \\
\text { [IQR] }\end{array}$ & Dosage & Duration & Main Outcome \\
\hline $\begin{array}{l}\text { The RECOVERY } \\
\text { Collaborative Group (2) }\end{array}$ & $324 / 689$ & $\mathrm{DX}$ & 8 [5-13] & 6 mg qd & $\begin{array}{l}\text { Up to } \\
10 \text { days }\end{array}$ & $\begin{array}{l}\text { The incidence of death is lower among patients } \\
\text { receiving IMV ( } 29.3 \% \text { vs. } 41.4 \% \text {; rate ratio, } 0.64 \text {; } \\
95 \% \mathrm{Cl}, 0.51 \text { to } 0.81)\end{array}$ \\
\hline Jeronimo et al. (66) & $194 / 199$ & MP & $13[9-16]$ & $0.5 \mathrm{mg} / \mathrm{kg}$ bid & 5 days & $\begin{array}{l}\text { The overall } 28 \text {-day mortality was not significant } \\
\text { between the placebo group and the MP group } \\
(\mathrm{P}=0.629)\end{array}$ \\
\hline $\begin{array}{l}\text { The Writing Committee } \\
\text { for the REMAP-CAP } \\
\text { Investigators (62) }\end{array}$ & $283 / 101$ & $\mathrm{HC}$ & N/A & $\begin{array}{l}50 \mathrm{mg} \text { or } 100 \mathrm{mg} \\
\text { q6h or } 50 \mathrm{mg} \text { q6h } \\
\text { when shock was } \\
\text { clinically evident }\end{array}$ & 7 days & $\begin{array}{l}\mathrm{HC} \text { resulted in } 93 \% \text { probabilities of superiority, } \\
\text { with regard to the odds of improvement in } \\
\text { organ support-free days within } 21 \text { days }\end{array}$ \\
\hline Dequin et al. (64) & $76 / 73$ & $\mathrm{HC}$ & N/A & $\begin{array}{l}200 \mathrm{mg} \text { qd then } \\
100 \mathrm{mg} \text { qd then } \\
50 \mathrm{mg} \text { qd }\end{array}$ & $\begin{array}{l}7 \text { days then } \\
4 \text { days then } \\
3 \text { days }\end{array}$ & $\begin{array}{l}\text { There was no significant difference for } \\
\text { Treatment failure on day } 21 \text { between } \mathrm{HC} \text { group } \\
\text { compared and the placebo group }(\mathrm{P}=0.29)\end{array}$ \\
\hline Edalatifard et al. (65) & $34 / 28$ & MP & $\mathrm{N} / \mathrm{A}$ & $250 \mathrm{mg}$ qd & 3 days & $\begin{array}{l}\text { The mortality rate was lower in the MP group } \\
(5.9 \% \text { versus } 42.9 \% \text {; } P<0 \cdot 001) \text {. Patients in the } \\
\text { MP group also had a significantly increased } \\
\text { survival time }(P<0.001)\end{array}$ \\
\hline Corral-Gudino et al. (63) & $56 / 29$ & MP & N/A & $\begin{array}{l}40 \mathrm{mg} \text { bid, then } \\
20 \mathrm{mg} \text { bid }\end{array}$ & $\begin{array}{l}3 \text { days then } \\
3 \text { days }\end{array}$ & $\begin{array}{l}\text { The use of MP was associated with a reduced } \\
\text { risk of the composite endpoint in the intention- } \\
\text { to-treat, age-stratified analysis }(\mathrm{P}=0.024)\end{array}$ \\
\hline
\end{tabular}

When different regimens were adopted in a study, the one that dominated is presented here. RCT, randomized clinical trial; DX, Dexamethasone; MP, Methylprednisolone; HC, Hydrocortisone; T, Testing group; C, Control group; GC, glucocorticoid; qd, quaque die; bid, bis in die; IMV, Invasive Mechanical Ventilation, IQR: interquartile range.

in many inflammatory diseases. But recommendations for systemic use of GC when treating severe infections, such as sepsis, are weak and are not well supported (55). In the previous SARS and Middle East respiratory syndrome (MERS) epidemics, while clinical evidence was inconclusive, GCs were still regarded as important treatment options (56-58). In this COVID-19 pandemic, the current evidence indicates that inflammation is a prominent pathophysiological process (59), with acute respiratory distress syndrome (ARDS) observed in over $70 \%$ of ICU patients and $90 \%$ of nonsurvivors (60). As GCs potentially improve patient outcomes in similar diseases such as ARDS (61), several studies have investigated its potential effects.

To date, seven RCTs (2,62-67) with substantially different GC administration timings, dosages, and durations have investigated whether GCs positively affect patients with severe COVID-19. Importantly, most have reported positive results, such as improved 28-day mortality (Table 1). A meta-analysis also concluded that GCs improved 28-day mortality (68). Based on these observations, clinical guidelines and protocols have been updated to recommend GCs for severely infected patients (69-71).

\section{Timing of GC administration}

The timing of GC administration is highly significant in affecting not only its pharmacological properties, but also disease course. Physiologically, early GC administration may be critical to decrease the acute and long-term negative impact on critically ill patients, as homeostatic correction could quickly turn into exhaustion $(31,72)$. Early $(<72 \mathrm{~h})$ methylprednisolone (MP) administration when compared to late ( $\geq 7$ days) was associated with faster disease resolution and ICU discharge in patients with ARDS (73). Recently, 
the DEXA-ARDS trial concluded that early dexamethasone administration reduced the duration of mechanical ventilation (MV) and overall mortality (74).

In patients with severe COVID-19, when RCTs reported GC timings $(2,66,67)$, the average time for treatment initiation was approximately $8-13$ days from symptom onset, a relatively late GC treatment schedule. Mixed results concerning mortality and days free from ventilation were reported in these studies. As benefits of early initiation of treatment have been proved in ARDS, an earlier treatment initiation is worthy of investigation. According to a recently published clinical-therapeutic staging proposal, an early phase in COVID-19 is characterized by an incubation period with mild and non-specific symptoms (75). For the moment, clinicians should decide on a case-by-case basis as no clear evidence has indicated the best possible initiation time for treatment. However, this kind of research is difficult. Confirming a COVID-19 diagnosis takes time, and hospitals are under huge pressure with unpredictable patient surges. Therefore, trialing and treating patients in an orderly manner are highly challenging.

\section{GC dosage}

While the precise relationship between GC dose, cellular concentration, and clinical effects remain to be established, it is believed an adequate initial loading bolus is required, particularly when GCs are administered as a continuous infusion (76). Tapering is also another key factor. RCTs have reported that abrupt GC discontinuation was rapidly followed by rebound inflammatory responses with severe clinical relapses (77-79). Additional MV days and increased morality risk were also reported $(80,81)$.

In most critical care studies, the daily MP equivalent of $80-100 \mathrm{mg}$ (dexamethasone $15-18.8 \mathrm{mg}$ ) is used to separate low from moderate doses (31). Low-to-moderate GC doses (MP $<2 \mathrm{mg} / \mathrm{kg} /$ day) significantly reduced the mortality rate of patients with ARDS, while high-GC doses (MP $>2 \mathrm{mg} / \mathrm{kg} /$ day) provided no significant benefit toward mortality rate reduction (82). In the aforementioned DEXA-ARDS trial, patients in the dexamethasone group received a daily intravenous dose of $20 \mathrm{mg}$ from day 1 to 5 , which was reduced to $10 \mathrm{mg}$ daily from day 6 to 10 . Reduced duration of mechanical ventilation and higher overall mortality in patients with established moderate-to severe ARDS were observed (74).

Despite the importance of an initial loading bolus, different dosage regimens have been reported in patients with severe COVID-19 (2,62-67). One particular regimen comprised consistent GC doses throughout the treatment course. However, mixed results were reported for this approach $(2,66)$. The administration of $6 \mathrm{mg}$ dexamethasone for up to 10 days reduced the 28-day mortality in those receiving either IMV or oxygen alone (2), while MP at $0.5 \mathrm{mg} / \mathrm{kg}$ for 5 days did not reduce mortality (66). Another regimen comprised the administration of tapering dosages, but inconsistent results were reported $(62-64,67)$. This dosage category did not reduce treatment failure in patients with COVID-19-related acute respiratory failure (64). However, it was associated with more ventilator-free days (67). Another regimen comprised the administration of pulse dosages for a short time period; an MP pulse (intravenous injection, $250 \mathrm{mg} /$ day for 3 days) generated a significantly increased survival time in patients with severe COVID-19. But the impact of this study is limited as a very small number of participants (34 patients in each group) were included and that ARDS was considered an exclusion criterion, thus offering poor values when treating patients with severe COVID-19. (65). While direct comparisons between studies are questionable, as certain variables were not controlled, these results suggest more studies are required to investigate the impact of GC dosage. Thus, clinical decisions should be made without excluding all options.

\section{GC duration}

The duration of GC administration is a main determinant of treatment efficacy (31). A protocol for prolonged MP treatment in patients with early ARDS, featuring a 28-day tapering plan, was recommended in recently published guidelines (83).

Durations adopted in RCTs for GC treatment in patients with severe COVID-19 are related to the dosage regimen being used and whether tapering was included. The only trial with pulse dosage treatment lasted for 3 days, with positive results observed (65). A consistent dosage plan was provided for 5,7 , and up to 10 days in different trials $(2,65,66)$, and tapering was included in three trials, lasting from 6-14 days. However, no consensus results were reached across categories.

These mixed timing, dosage and duration results were attributed to several reasons. Both inclusion criteria and patient baseline characteristics differed greatly between studies. Patient populations with different mean ages, different disease stages, and a wide range of diverse 
commodities were investigated. These factors may have had a significant impact on treatment outcomes. In addition, positive conclusions may be associated with the selection of correct patient groups. In the RECOVERY study, mortality benefits were only observed in patients receiving IMV or oxygen alone (2). Many other studies have adopted partial pressure of oxygen $\left(\mathrm{PaO}_{2}\right) /$ fraction of inspiration $\mathrm{O}_{2}\left(\mathrm{FiO}_{2}\right)$ in their inclusion criteria. However, targeting this particular physiological marker may not be enough to filter potential groups who could likely benefit from GCs, whatever the regimen is. Finally, systemic variations, such as differences between healthcare systems and practice norms may also have an impact.

Despite considerable efforts, it is extremely difficult to derive definitive conclusions on GC timing, dosage, and duration from the literature. Firstly, completed RCTs still focus on the effectiveness of GCs in general rather than specific regimens during treatment. Also, GC type, timing, dosage, and duration vary greatly between studies, making it difficult to draw conclusions due a lack of high-quality evidence. Lastly, some RCTs (64) completed early due to issues with patient recruitment and the publication of results (e.g., RECOVERY), thereby questioning the strength of study outcomes.

At the same time, potential severe adverse effects associated with GCs need to be considered and cost-benefit analysis should be made when substantial uncertainty occurs. Traditionally, long-term use of GC is associated with various complications, such as infections, diabetes and osteoporosis, psychiatric disorders, and adrenal crisis $(84,85)$. High-dose GCs is related to many metabolic disorders, such as hypokalemia and intravenous pulse GCs have been associated with hypotension, electrolyte disorders, anaphylactic shock, and abnormal behavior (86). For patients with severe COVID-19, one systemic review including 6 trials concluded that there was no suggestion that the risk of serious adverse events was higher in patients treated with GCs except for the 2 smallest trials (68). A further systemic review published recently draw the conclusion that there were unclear differences in rates of neuromuscular weakness and gastrointestinal bleeding with GCs. Increase in superinfection was not observed. But there was probably an increase in hyperglycemia (87).

Unequivocally, GC benefits in patients with severe COVID-19 are dependent on the selection of the right dose, at the right time, in the right patient group. Using new methods to target specific patient groups, guiding GC treatments based on disease progression, and evaluating treatment effectiveness should be key research objectives in future research.

\section{Tailoring GC treatments}

GC regimens lasting 7-10 days have been recommended in updated $\mathrm{WHO}$ guidelines for treating patients with severe COVID-19 (3), but the exact timing, duration, and dosage remain, and whether proper weaning should be considered unclear. As more clinical trials are being conducted, it appears that in a short period of time, the concept of "one regimen fits all" cannot be adequately addressed in current RCT models.

Previously, it was reported that patients with ARDS receiving similar GC doses experienced a substantial variability in plasma concentrations due to betweenpatient variability, plus additional disease effects from GC pharmacokinetics were also observed, potentially affecting clinical responses (88). Therefore, tailored dosages and therapy durations based on individual patient responses are essential.

Undoubtedly, current clinical research has generated beneficial parameters to evaluate end-point incidents (2,62-67). Several RCTs and observational studies have adopted mortality rates and ventilation status as primary or secondary outcomes $(2,65,67)$. However, a lack of evaluation and assessment tools during disease progression means physicians are less likely to adequately assess ambulatory characteristics and decide when, how much, and how long GCs should be administered.

Several biological markers have been adopted to reflect disease severity and may help tailor GC treatments. Of these, C-reactive protein (CRP) (89-91), lactate dehydrogenase (LDH) $(92,93)$, neutrophil lymphocyte ratios $(94)$, and D-dimers $(90,95,96)$ alone are all associated with disease progression severity. Other markers, such as the interleukins (97), ferritin $(98,99)$, and several cardiac markers (100) have also been explored. In one study, a combination of two markers (neutrophil-to-lymphocyte ratio and $\mathrm{CRP}$ ) correlated with disease severity, similar to individual use (101). For patients with elevated CRP, GC treatment was linked to reduced mortality risk or MV (102), suggesting this marker may help select patients benefitting from GCs. However, several limitations to this approach must be considered. Firstly, as several biomarkers reflect disease severity, with no consensus on superiority, it is difficult for clinicians to decide on the importance of one marker and associated changes during infection. 


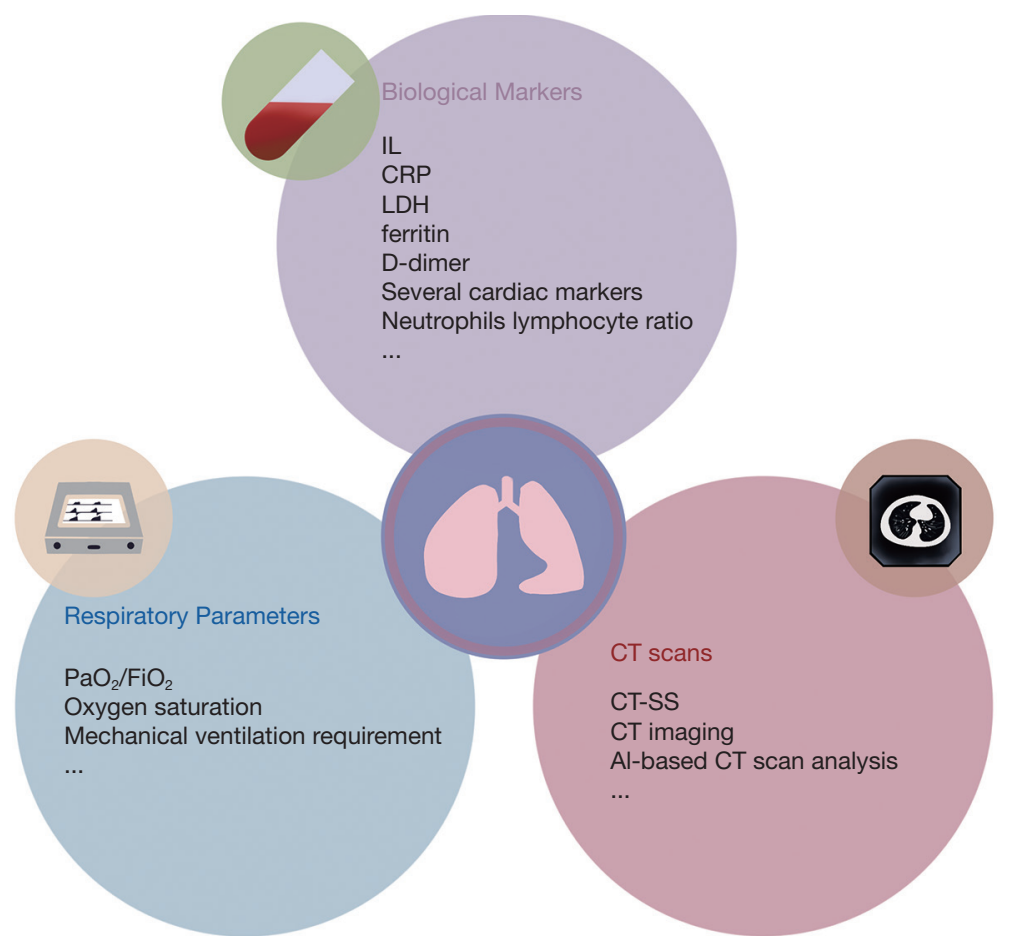

Figure 2 Three dimensions of tailoring glucocorticoids. The efficacy of glucocorticoid (GC) treatment can be monitored by taking three perspectives, biological markers, respiratory parameters and radiological evidence into consideration. CRP, C-reactive protein; LDH, lactate dehydrogenase; IL, Interleukin; $\mathrm{PaO}_{2}$, partial arterial pressure of oxygen; $\mathrm{FiO}_{2}$, fraction of inspiration oxygen; CT, computed tomography; CT-SS computed tomography severity score, AI, artificial intelligence.

Additionally, biological markers tend to be influenced by a number of factors, e.g., CRP is elevated during multiple inflammatory conditions, including rheumatoid arthritis and some cardiovascular diseases, besides COVID-19 (103). Thus, more studies are required to investigate the potential impact of these markers on COVID-19.

Respiratory parameters could also be used to define severe COVID-19 infections. In general, such parameters are defined by at least one of the following characteristics: respiratory distress ( $\geq 30$ times/min), oxygen saturation $\leq 93 \%$ at rest, respiratory failure requiring $\mathrm{MV}$, and $\mathrm{PaO}_{2} /$ $\mathrm{FiO}_{2} \leq 300 \mathrm{mmHg}(62,104-106)$. The use of GCs for patient receiving IMV resulted in a lower 28-day mortality (2), suggesting that respiratory parameters could be adopted to select patients for GC treatment. However, clinical hazards must be indicated if these parameters are used. Undesirable changes in $\mathrm{PaO}_{2} / \mathrm{FiO}_{2}$ or oxygen saturation levels in patients on oxygen therapies do not necessarily reflect progression of an initial infection. Ventilator-induced lung injury caused by inappropriate tidal volume or positive end-expiratory pressure could worsen the ventilation status of patients
$(104,107)$. Other complications, such as ventilator-related infections by bacteria, may also affect respiratory status (108).

In addition, most clinical trials $(2,62-64,67)$ have included inflammatory markers and ventilation status to their study designs, but the benefit of adding radiological evidence to guide GC treatment is warranted (Figure 2).

Chest computed tomography (CT) has been used in the diagnosis and evaluation of many respiratory diseases, such as pneumonia (109) and interstitial lung disease (110). It is accepted that monitoring responses to GC treatment during intervention, using daily assessments of lung, multiple organ function, and systemic inflammation marker measurements are essential $(31,111)$.

Lung infection severity may be assessed using CT imaging in a comparatively objective manner. A chest CT severity score (CT-SS) has been proposed to evaluate patient severity upon diagnosis, and importantly provides crucial prognostic information. The score uses lung opacification as a surrogate for extension of the disease, and could be used to rapidly and objectively evaluate the severity of pulmonary involvement (112). Also, these scores 
have implications for predicting the progression risk of patients with COVID-19 pneumonia at admission, riskstratification, and admission timing (113).

CT not only offers crucial information on lung pathology, but is also a good indicator of systemic characteristics. CTSS is positively associated with several inflammatory indices (e.g., neutrophil counts, LDH, and CRP) and negatively associated with lymphocyte counts $(114,115)$. Thus, CT-SS provides considerable morphological information on lung inflammation progression as well as systemic inflammatory status.

Modern technology may help increase evaluation accuracy based on CT analyses. Artificial Intelligence (AI)guided severity assessments and patient-following could have promising roles in guiding GC treatments. Thus, AI and CT could help precisely evaluate COVID-19 pneumonia severity and also patient clinical surveillance (116). As the role of AI in patient diagnostics has already been confirmed (117), its combination with CT-SS as an AIguided GC treatment could offer more precise clinical options for clinicians.

In their study, Su et al. reported several cases where AIbased CT scans were used to adjust GC parameters (dosage and duration) in patients, with the authors concluding that sufficient GCs may be effective in treating patients with COVID-19, concomitant with frequent evaluation and timely adjustment (118). However, further research is required to investigate how GCs may be adjusted based on a combinatorial approach of physiological markers, laboratory results, and CT analysis. Similarly, limitations to this approach exist, e.g., appropriate algorithms must be tested to objectively and precisely reflect lung opacification. More importantly, in hospitals with limited capacity to separate patients, the risk of spreading disease while transferring patients should also be considered. For patients, frequent CT scans may generate radiation exposure risks, and treatment costs not covered by healthcare systems may generate increased economic burdens to patients outside these systems. Lastly, these approaches are limited to facilities where CT technologies are readily available.

\section{Conclusions}

GCs play key roles in treating severe COVID-19 infections. We investigated the unique properties of GCs toward immune cell modulation, cytokine reduction, and improved endothelial function, which reflect the major pathophysiological processes induced by COVID-19.
The clinical studies reviewed here have somewhat proven the efficacy of GCs in patients with severe COVID-19. However, timing, dosage, and duration varied considerably among studies. GC treatment initiation is relatively late when compared with ARDS, with a tendency to administer low-to-moderate GC doses. Importantly, tapering as an administrative method is not widely accepted. Thus, early treatment initiation and a tailored dosage with appropriate tapering may be of particular relevance. Equally, biological markers, respiratory parameters, and radiological evidence may be used in a combinatorial manner to tailor GC treatments to patients with COVID-19.

\section{Acknowledgments}

Funding: This article was supported by grants from Natural Science Foundation of Shanghai (20ZR1411100), Program of Shanghai Academic/Technology Research Leader (20XD1421000), National Natural Science Foundation of China (82070085), the Research Funds of Shanghai Municipal Health Commission (2019ZB0105), Clinical Research Funds of Zhongshan Hospital (2020ZSLC38 and 2020ZSLC27), Smart Medical Care of Zhongshan Hospital (2020ZHZS01) and Science and Technology Commission of Shanghai Municipality (20DZ2261200)

\section{Footnote}

Reporting Checklist: The authors have completed the Narrative Review reporting checklist. Available at https:// dx.doi.org/10.21037/atm-21-1783

Peer Review File: Available at https://dx.doi.org/10.21037/ atm-21-1783

Conflicts of Interest: All authors have completed the ICMJE uniform disclosure form (available at https://dx.doi. org/10.21037/atm-21-1783). Dr. GWT reports grants from National Natural Science Foundation of China (82070085), Program of Shanghai Academic/Technology Research Leader (20XD1421000) and Clinical Research Funds of Zhongshan Hospital (2020ZSLC38). Dr. ZL reports grants from Clinical Research Funds of Zhongshan Hospital Funding (2020ZSLC27), Smart Medical Care of Zhongshan Hospital Funding (2020ZHZS01), Natural Science Foundation of Shanghai (20ZR1411100), The Research Funds of Shanghai Municipal Health Commission (2019ZB0105) and Science and Technology Commission of 
Shanghai Municipality (20DZ2261200). The other authors have no conflicts of interest to declare.

Ethical Statement: The authors are accountable for all aspects of the work in ensuring that questions related to the accuracy or integrity of any part of the work are appropriately investigated and resolved.

Open Access Statement: This is an Open Access article distributed in accordance with the Creative Commons Attribution-NonCommercial-NoDerivs 4.0 International License (CC BY-NC-ND 4.0), which permits the noncommercial replication and distribution of the article with the strict proviso that no changes or edits are made and the original work is properly cited (including links to both the formal publication through the relevant DOI and the license). See: https://creativecommons.org/licenses/by-nc-nd/4.0/.

\section{References}

1. WHO Coronavirus (COVID-19) Dashboard. Available online: https://covid19.who.int/?gclid=Cj0KCQiAvvKB BhCXARIsACTePW8fiUVEK-PdhTlv3fMIW0BAo3ju_ PRRgddMBh1wfI-YN9-e9vcO22saAtPqEALw_wcB

2. RECOVERY Collaborative Group; Horby P, Lim WS, et al. Dexamethasone in Hospitalized Patients with Covid-19. N Engl J Med 2021;384:693-704.

3. Rochwerg B, Siemieniuk RA, Agoritsas T, et al. A living WHO guideline on drugs for covid-19. BMJ 2020;370:m3379.

4. Tay MZ, Poh CM, Rénia L, et al. The trinity of COVID-19: immunity, inflammation and intervention. Nat Rev Immunol 2020;20:363-74.

5. Satturwar S, Fowkes M, Farver C, et al. Postmortem Findings Associated With SARS-CoV-2: Systematic Review and Meta-analysis. Am J Surg Pathol 2021;45:587-603.

6. Zarrilli G, Angerilli V, Businello G, et al. The Immunopathological and Histological Landscape of COVID-19-Mediated Lung Injury. Int J Mol Sci 2021;22:974.

7. Bidari Zerehpoosh F, Sabeti S, Bahrami-Motlagh H, et al. Post-mortem Histopathologic Findings of Vital Organs in Critically Ill Patients with COVID-19. Arch Iran Med 2021;24:144-51.

8. Kim JS, Lee JY, Yang JW, et al. Immunopathogenesis and treatment of cytokine storm in COVID-19. Theranostics 2021;11:316-29.
9. Soy M, Keser G, Atagündüz P, et al. Cytokine storm in COVID-19: pathogenesis and overview of antiinflammatory agents used in treatment. Clin Rheumatol 2020;39:2085-94.

10. Tang Y, Liu J, Zhang D, et al. Cytokine Storm in COVID-19: The Current Evidence and Treatment Strategies. Front Immunol 2020;11:1708.

11. Huang C, Wang Y, Li X, et al. Clinical features of patients infected with 2019 novel coronavirus in Wuhan, China. Lancet 2020;395:497-506.

12. Fajgenbaum DC, June CH, et al. Cytokine Storm. N Engl J Med 2020;383:2255-73.

13. Zhang J, Hao Y, Ou W, et al. Serum interleukin-6 is an indicator for severity in 901 patients with SARS-CoV-2 infection: a cohort study. J Transl Med 2020;18:406.

14. Bösmüller H, Matter M, Fend F, et al. The pulmonary pathology of COVID-19. Virchows Arch 2021;478:137-50.

15. Bonaventura A, Vecchié A, Dagna L, et al. Endothelial dysfunction and immunothrombosis as key pathogenic mechanisms in COVID-19. Nat Rev Immunol 2021;21:319-29.

16. Gu SX, Tyagi T, Jain K, et al. Thrombocytopathy and endotheliopathy: crucial contributors to COVID-19 thromboinflammation. Nat Rev Cardiol 2021;18:194-209.

17. Mosleh W, Chen K, Pfau SE, et al. Endotheliitis and Endothelial Dysfunction in Patients with COVID-19: Its Role in Thrombosis and Adverse Outcomes. J Clin Med 2020. [Epub ahead of print] doi:10.3390/jcm9061862.

18. Menezes MCS, Pestana DVS, Gameiro GR, et al. SARS-CoV-2 pneumonia-receptor binding and lung immunopathology: a narrative review. Crit Care 2021;25:53.

19. Xu Z, Shi L, Wang Y, et al. Pathological findings of COVID-19 associated with acute respiratory distress syndrome. Lancet Respir Med 2020;8:420-2.

20. Goshua G, Pine AB, Meizlish ML, et al. Endotheliopathy in COVID-19-associated coagulopathy: evidence from a single-centre, cross-sectional study. Lancet Haematol 2020;7:e575-82.

21. Cabbab ILN, Manalo RVM, et al. Anti-inflammatory drugs and the renin-angiotensin-aldosterone system: Current knowledge and potential effects on early SARSCoV-2 infection. Virus Res 2021;291:198190.

22. Saba L, Gerosa C, Fanni D, et al. Molecular pathways triggered by COVID-19 in different organs: ACE2 receptor-expressing cells under attack? A review. Eur Rev Med Pharmacol Sci 2020;24:12609-22.

23. Tikellis C, Thomas MC, et al. Angiotensin-Converting 
Enzyme 2 (ACE2) Is a Key Modulator of the Renin Angiotensin System in Health and Disease. Int J Pept 2012;2012:256294.

24. Wolf G, Wenzel U, Burns KD, et al. Angiotensin II activates nuclear transcription factor-kappaB through AT1 and AT2 receptors. Kidney Int 2002;61:1986-95.

25. Hariharan A, Hakeem AR, Radhakrishnan S, et al. The Role and Therapeutic Potential of NF-kappa-B Pathway in Severe COVID-19 Patients. Inflammopharmacology 2021;29:91-100.

26. Hirano T, Murakami M, et al. COVID-19: A New Virus, but a Familiar Receptor and Cytokine Release Syndrome. Immunity 2020;52:731-3.

27. Han Y, Runge MS, Brasier AR, et al. Angiotensin II induces interleukin-6 transcription in vascular smooth muscle cells through pleiotropic activation of nuclear factor-kappa B transcription factors. Circ Res 1999;84:695-703.

28. Zhang Q, Lenardo MJ, Baltimore D, et al. 30 Years of NF$\kappa \mathrm{B}$ : A Blossoming of Relevance to Human Pathobiology. Cell 2017;168:37-57

29. Timmermans S, Souffriau J, Libert C, et al. A General Introduction to Glucocorticoid Biology. Front Immunol 2019;10:1545.

30. Pratt WB, Toft DO, et al. Regulation of signaling protein function and trafficking by the hsp90/hsp70based chaperone machinery. Exp Biol Med (Maywood) 2003;228:111-33.

31. Meduri GU, Annane D, Confalonieri M, et al. Pharmacological principles guiding prolonged glucocorticoid treatment in ARDS. Intensive Care Med 2020;46:2284-96.

32. Meijsing SH, Pufall MA, So AY, et al. DNA binding site sequence directs glucocorticoid receptor structure and activity. Science 2009;324:407-10.

33. Baschant U, Tuckermann J, et al. The role of the glucocorticoid receptor in inflammation and immunity. J Steroid Biochem Mol Biol 2010;120:69-75.

34. Ehrchen JM, Roth J, Barczyk-Kahlert K, et al. More Than Suppression: Glucocorticoid Action on Monocytes and Macrophages. Front Immunol 2019;10:2028.

35. Petrillo MG, Bortner CD, Cidlowski JA. Glucocorticoids: Inflammation and Immunity. In: Geer EB. The Hypothalamic-Pituitary-Adrenal Axis in Health and Disease. Switzerland: Springer International Publishing, 2017:43-63.

36. Liberman AC, Budziñski ML, Sokn C, et al. Regulatory and Mechanistic Actions of Glucocorticoids on $\mathrm{T}$ and
Inflammatory Cells. Front Endocrinol (Lausanne) 2018;9:235.

37. Besedovsky L, Born J, Lange T, et al. Endogenous glucocorticoid receptor signaling drives rhythmic changes in human T-cell subset numbers and the expression of the chemokine receptor CXCR4. FASEB J 2014;28:67-75.

38. Fischer HJ, Schweingruber N, Lühder F, et al. The potential role of $\mathrm{T}$ cell migration and chemotaxis as targets of glucocorticoids in multiple sclerosis and experimental autoimmune encephalomyelitis. Mol Cell Endocrinol 2013;380:99-107.

39. Ugor E, Prenek L, Pap R, et al. Glucocorticoid hormone treatment enhances the cytokine production of regulatory $T$ cells by upregulation of Foxp3 expression. Immunobiology 2018;223:422-31.

40. Karagiannidis C, Akdis M, Holopainen P, et al. Glucocorticoids upregulate FOXP3 expression and regulatory $\mathrm{T}$ cells in asthma. J Allergy Clin Immunol 2004;114:1425-33.

41. Wan YY. Regulatory T cells: immune suppression and beyond. Cell Mol Immunol 2010;7:204-10.

42. Barnes PJ. Anti-inflammatory actions of glucocorticoids: molecular mechanisms. Clin Sci (Lond) 1998;94:557-72.

43. Amano Y, Lee SW, Allison AC, et al. Inhibition by glucocorticoids of the formation of interleukin-1 alpha, interleukin-1 beta, and interleukin-6: mediation by decreased mRNA stability. Mol Pharmacol 1993;43:176-82.

44. Coutinho AE, Chapman KE, et al. The anti-inflammatory and immunosuppressive effects of glucocorticoids, recent developments and mechanistic insights. Mol Cell Endocrinol 2011;335:2-13.

45. Auphan N, DiDonato JA, Rosette C, et al. Immunosuppression by glucocorticoids: inhibition of NFkappa B activity through induction of I kappa B synthesis. Science 1995;270:286-90.

46. Perico L, Benigni A, Casiraghi F, et al. Immunity, endothelial injury and complement-induced coagulopathy in COVID-19. Nat Rev Nephrol 2021;17:46-64.

47. Zakkar M, Luong le A, Chaudhury H, et al. Dexamethasone arterializes venous endothelial cells by inducing mitogen-activated protein kinase phosphatase-1: a novel antiinflammatory treatment for vein grafts? Circulation 2011;123:524-32.

48. Vettorazzi S, Bode C, Dejager L, et al. Glucocorticoids limit acute lung inflammation in concert with inflammatory stimuli by induction of SphK1. Nat Commun 2015;6:7796.

49. McVerry BJ, Peng X, Hassoun PM, et al. Sphingosine 1 -phosphate reduces vascular leak in murine and canine 
models of acute lung injury. Am J Respir Crit Care Med 2004;170:987-93.

50. Förster C, Burek M, Romero IA, et al. Differential effects of hydrocortisone and TNFalpha on tight junction proteins in an in vitro model of the human blood-brain barrier. J Physiol 2008;586:1937-49.

51. Cui N, Wang H, Long Y, et al. Dexamethasone Suppressed LPS-Induced Matrix Metalloproteinase and Its Effect on Endothelial Glycocalyx Shedding. Mediators Inflamm 2015;2015:912726.

52. Blecharz KG, Haghikia A, Stasiolek M, et al. Glucocorticoid effects on endothelial barrier function in the murine brain endothelial cell line cEND incubated with sera from patients with multiple sclerosis. Mult Scler 2010;16:293-302.

53. Förster C, Kahles T, Kietz S, et al. Dexamethasone induces the expression of metalloproteinase inhibitor TIMP-1 in the murine cerebral vascular endothelial cell line cEND. J Physiol 2007;580:937-49.

54. Zieli ska KA, Van Moortel L, Opdenakker G, et al. Endothelial Response to Glucocorticoids in Inflammatory Diseases. Front Immunol 2016;7:592.

55. Rhodes A, Evans LE, Alhazzani W, et al. Surviving Sepsis Campaign: International Guidelines for Management of Sepsis and Septic Shock: 2016. Crit Care Med 2017;45:486-552.

56. Lee KH, Yoon S, Jeong GH, et al. Efficacy of Corticosteroids in Patients with SARS, MERS and COVID-19: A Systematic Review and Meta-Analysis. J Clin Med 2020;9:2392.

57. Li H, Chen C, Hu F, et al. Impact of corticosteroid therapy on outcomes of persons with SARS-CoV-2, SARS$\mathrm{CoV}$, or MERS-CoV infection: a systematic review and meta-analysis. Leukemia 2020;34:1503-11.

58. Panesar NS. Glucocorticoid treatment of patients with SARS: implications for mechanisms of immunopathology. Nat Rev Immunol 2006;6:334.

59. Mahmudpour M, Roozbeh J, Keshavarz M, et al. COVID-19 cytokine storm: The anger of inflammation. Cytokine 2020;133:155151.

60. Tzotzos SJ, Fischer B, Fischer H, et al. Incidence of ARDS and outcomes in hospitalized patients with COVID-19: a global literature survey. Crit Care 2020;24:516.

61. Meduri GU, Siemieniuk RAC, Ness RA, et al. Prolonged low-dose methylprednisolone treatment is highly effective in reducing duration of mechanical ventilation and mortality in patients with ARDS. J Intensive Care 2018;6:53.
62. Angus DC, Derde L, Al-Beidh F, et al. Effect of Hydrocortisone on Mortality and Organ Support in Patients With Severe COVID-19: The REMAP-CAP COVID-19 Corticosteroid Domain Randomized Clinical Trial. JAMA 2020;324:1317-29.

63. Corral-Gudino L, Bahamonde A, Arnaiz-Revillas F, et al. GLUCOCOVID: A controlled trial of methylprednisolone in adults hospitalized with COVID-19 pneumonia. medRxiv 2020.06.17.20133579; doi: https://doi.org/10.110 1/2020.06.17.20133579.

64. Dequin PF, Heming N, Meziani F, et al. Effect of Hydrocortisone on 21-Day Mortality or Respiratory Support Among Critically Ill Patients With COVID-19: A Randomized Clinical Trial. JAMA 2020;324:1298-306.

65. Edalatifard M, Akhtari M, Salehi M, et al. Intravenous methylprednisolone pulse as a treatment for hospitalised severe COVID-19 patients: results from a randomised controlled clinical trial. Eur Respir J 2020;56:2002808.

66. Jeronimo CMP, Farias MEL, Val FFA, et al. Methylprednisolone as Adjunctive Therapy for Patients Hospitalized With Coronavirus Disease 2019 (COVID-19; Metcovid): A Randomized, Double-blind, Phase IIb, Placebo-controlled Trial. Clin Infect Dis 2021;72:e373-81.

67. Tomazini BM, Maia IS, Cavalcanti AB, et al. Effect of Dexamethasone on Days Alive and Ventilator-Free in Patients With Moderate or Severe Acute Respiratory Distress Syndrome and COVID-19: The CoDEX Randomized Clinical Trial. JAMA 2020;324:1307-16.

68. WHO Rapid Evidence Appraisal for COVID-19 Therapies (REACT) Working Group; Sterne JAC, Murthy S, et al. Association Between Administration of Systemic Corticosteroids and Mortality Among Critically Ill Patients With COVID-19: A Meta-analysis. JAMA 2020;324:1330-41.

69. Therapeutic Management of Adults With COVID-19. Available online: https://www.covid19treatmentguidelines. nih.gov/therapeutic-management/

70. Corticosteroids for COVID-19. Available online: https:// www.who.int/publications/i/item/WHO-2019-nCoVCorticosteroids-2020.1

71. China NHCotPsRo, Diagnosis and Treatment of COVID-19 Pneumonia (8th edition). Infectious Disease Information

72. Meduri GU, Chrousos GP, et al. General Adaptation in Critical Illness: Glucocorticoid Receptor-alpha Master Regulator of Homeostatic Corrections. Front Endocrinol (Lausanne) 2020;11:161.

73. Meduri GU, Bridges L, Shih MC, et al. Prolonged 
glucocorticoid treatment is associated with improved ARDS outcomes: analysis of individual patients' data from four randomized trials and trial-level meta-analysis of the updated literature. Intensive Care Med 2016;42:829-40.

74. Villar J, Ferrando C, Martínez D, et al. Dexamethasone treatment for the acute respiratory distress syndrome: a multicentre, randomised controlled trial. Lancet Respir Med 2020;8:267-76.

75. Siddiqi HK, Mehra MR, et al. COVID-19 illness in native and immunosuppressed states: A clinical-therapeutic staging proposal. J Heart Lung Transplant 2020;39:405-7.

76. Buttgereit F, da Silva JA, Boers M, et al. Standardised nomenclature for glucocorticoid dosages and glucocorticoid treatment regimens: current questions and tentative answers in rheumatology. Ann Rheum Dis 2002;61:718-22.

77. Keh D, Boehnke T, Weber-Cartens S, et al. Immunologic and hemodynamic effects of "low-dose" hydrocortisone in septic shock: a double-blind, randomized, placebocontrolled, crossover study. Am J Respir Crit Care Med 2003;167:512-20.

78. Gagnon S, Boota AM, Fischl MA, et al. Corticosteroids as adjunctive therapy for severe Pneumocystis carinii pneumonia in the acquired immunodeficiency syndrome. A double-blind, placebo-controlled trial. N Engl J Med 1990;323:1444-50.

79. Nawab QU, Golden E, Confalonieri M, et al. Corticosteroid treatment in severe community-acquired pneumonia: duration of treatment affects control of systemic inflammation and clinical improvement. Intensive Care Med 2011;37:1553-4.

80. Steinberg KP, Hudson LD, Goodman RB, et al. Efficacy and safety of corticosteroids for persistent acute respiratory distress syndrome. N Engl J Med 2006;354:1671-84.

81. Meduri GU, Bridges L, Siemieniuk RAC, et al. An Exploratory Reanalysis of the Randomized Trial on Efficacy of Corticosteroids as Rescue Therapy for the Late Phase of Acute Respiratory Distress Syndrome. Crit Care Med 2018;46:884-91.

82. Sun S, Liu D, Zhang H, et al. Effect of different doses and time-courses of corticosteroid treatment in patients with acute respiratory distress syndrome: A meta-analysis. Exp Ther Med 2019;18:4637-44.

83. Annane D, Pastores SM, Arlt W, et al. Critical IllnessRelated Corticosteroid Insufficiency (CIRCI): A Narrative Review from a Multispecialty Task Force of the Society of Critical Care Medicine (SCCM) and the European Society of Intensive Care Medicine (ESICM). Crit Care Med
2017;45:2089-98.

84. Grennan D, Wang S, et al. Steroid Side Effects. JAMA 2019;322:282.

85. Volmer T, Effenberger T, Trautner C, et al. Consequences of long-term oral corticosteroid therapy and its side-effects in severe asthma in adults: a focused review of the impact data in the literature. Eur Respir J 2018;52:1800703.

86. Bonnotte B, Chauffert B, Martin F, et al. Side-effects of high-dose intravenous (pulse) methylprednisolone therapy cured by potassium infusion. Br J Rheumatol 1998;37:109.

87. Chaudhuri D, Sasaki K, Karkar A, et al. Corticosteroids in COVID-19 and non-COVID-19 ARDS: a systematic review and meta-analysis. Intensive Care Med 2021;47:521-37.

88. Yates CR, Vysokanov A, Mukherjee A, et al. Time-variant increase in methylprednisolone clearance in patients with acute respiratory distress syndrome: a population pharmacokinetic study. J Clin Pharmacol 2001;41:415-24.

89. Ahnach M, Zbiri S, Nejjari S, et al. C-reactive protein as an early predictor of COVID-19 severity. J Med Biochem 2020;39:500-7.

90. Hodges G, Pallisgaard J, Schjerning Olsen AM, et al. Association between biomarkers and COVID-19 severity and mortality: a nationwide Danish cohort study. BMJ Open 2020;10:e041295.

91. Chen W, Zheng KI, Liu S, et al. Plasma CRP level is positively associated with the severity of COVID-19. Ann Clin Microbiol Antimicrob 2020;19:18.

92. Chen Z, Zhang F, Hu W, et al. Laboratory markers associated with COVID-19 progression in patients with or without comorbidity: A retrospective study. J Clin Lab Anal 2021;35:e23644.

93. Henry BM, Aggarwal G, Wong J, et al. Lactate dehydrogenase levels predict coronavirus disease 2019 (COVID-19) severity and mortality: A pooled analysis. Am J Emerg Med 2020;38:1722-6.

94. Li Y, Hou H, Diao J, et al. Neutrophil-to-lymphocyte ratio is independently associated with COVID-19 severity: An updated meta-analysis based on adjusted effect estimates. Int J Lab Hematol 2021. [Online ahead of print] doi:10.1111/ijlh.13475.

95. Du WN, Zhang Y, Yu Y, et al. D-dimer levels is associated with severe COVID-19 infections: A meta-analysis. Int J Clin Pract 2021:e14031.

96. Paliogiannis P, Mangoni AA, Dettori P, et al. D-Dimer Concentrations and COVID-19 Severity: A Systematic Review and Meta-Analysis. Front Public Health 
2020;8:432.

97. Dhar SK, K V, Damodar S, et al. IL-6 and IL-10 as predictors of disease severity in COVID-19 patients: results from meta-analysis and regression. Heliyon 2021;7:e06155.

98. Ahmed S, Ansar Ahmed Z, Siddiqui I, et al. Evaluation of serum ferritin for prediction of severity and mortality in COVID-19- A cross sectional study. Ann Med Surg (Lond) 2021;63:102163.

99. Velavan TP, Meyer CG, et al. Mild versus severe COVID-19: Laboratory markers. Int J Infect Dis 2020;95:304-7.

100. From the American Association of Neurological Surgeons (AANS), American Society of Neuroradiology (ASNR), Cardiovascular and Interventional Radiology Society of Europe (CIRSE), Canadian Interventional Radiology Association (CIRA), Congress of Neurological Surgeons (CNS), European Society of Minimally Invasive Neurological Therapy (ESMINT), European Society of Neuroradiology (ESNR), European Stroke Organization (ESO), Society for Cardiovascular Angiography and Interventions (SCAI), Society of Interventional Radiology (SIR), Society of NeuroInterventional Surgery (SNIS), and World Stroke Organization (WSO); Sacks D, Baxter B, et al. Multisociety Consensus Quality Improvement Revised Consensus Statement for Endovascular Therapy of Acute Ischemic Stroke. Int J Stroke 2018;13:612-32.

101.Liu YP, Li GM, He J, et al. Combined use of the neutrophil-to-lymphocyte ratio and CRP to predict 7-day disease severity in 84 hospitalized patients with COVID-19 pneumonia: a retrospective cohort study. Ann Transl Med 2020;8:635.

102. Keller MJ, Kitsis EA, Arora S, et al. Effect of Systemic Glucocorticoids on Mortality or Mechanical Ventilation in Patients With COVID-19. J Hosp Med 2020;15:489-93.

103. Du Clos TW, Mold C, et al. C-reactive protein: an activator of innate immunity and a modulator of adaptive immunity. Immunol Res 2004;30:261-77.

104. Attaway AH, Scheraga RG, Bhimraj A, et al. Severe covid-19 pneumonia: pathogenesis and clinical management. BMJ 2021;372:n436.

105. Berlin DA, Gulick RM, Martinez FJ, et al. Severe Covid-19. N Engl J Med 2020;383:2451-60.

106. Qin YY, Zhou YH, Lu YQ, et al. Effectiveness of glucocorticoid therapy in patients with severe coronavirus disease 2019: protocol of a randomized controlled trial. Chin Med J (Engl) 2020;133:1080-6.
107. Slutsky AS, Ranieri VM, et al. Ventilator-induced lung injury. N Engl J Med 2013;369:2126-36.

108. Hua J, Qian C, Luo Z, et al. Invasive mechanical ventilation in COVID-19 patient management: the experience with 469 patients in Wuhan. Crit Care 2020;24:348.

109. Garin N, Marti C, Carballo S, et al. Rational Use of CT-Scan for the Diagnosis of Pneumonia: Comparative Accuracy of Different Strategies. J Clin Med 2019. [Epub ahead of print] doi:10.3390/jcm8040514.

110. Bergin CJ, Müller NL, et al. CT of interstitial lung disease: a diagnostic approach. AJR Am J Roentgenol 1987;148:9-15.

111. Murray JF, Matthay MA, Luce JM, et al. An expanded definition of the adult respiratory distress syndrome. Am Rev Respir Dis 1988;138:720-3.

112. Yang R, Li X, Liu H, et al. Chest CT Severity Score: An Imaging Tool for Assessing Severe COVID-19. Radiol Cardiothorac Imaging 2020;2:e200047.

113.Feng Z, Yu Q, Yao S, et al. Early prediction of disease progression in COVID-19 pneumonia patients with chest CT and clinical characteristics. Nat Commun 2020;11:4968.

114. Saeed GA, Gaba W, Shah A, et al. Correlation between Chest CT Severity Scores and the Clinical Parameters of Adult Patients with COVID-19 Pneumonia. Radiol Res Pract 2021;2021:6697677.

115. Francone M, Iafrate F, Masci GM, et al. Chest CT score in COVID-19 patients: correlation with disease severity and short-term prognosis. Eur Radiol 2020;30:6808-17.

116. Carvalho ARS, Guimarães A, Werberich GM, et al. COVID-19 Chest Computed Tomography to Stratify Severity and Disease Extension by Artificial Neural Network Computer-Aided Diagnosis. Front Med (Lausanne) 2020;7:577609.

117. Khatami F, Saatchi M, Zadeh SST, et al. A meta-analysis of accuracy and sensitivity of chest CT and RT-PCR in COVID-19 diagnosis. Sci Rep 2020;10:22402.

118.Su Y, Han Y, Liu J, et al. Tailoring steroids in the treatment of COVID-19 pneumonia assisted by CT scans: three case reports. J Xray Sci Technol 2020;28:885-92.

Cite this article as: Luo MH, Qian YQ, Huang DL, Luo JC, Su Y, Wang H, Yu SJ, Liu K, Tu GW, Luo Z. Tailoring glucocorticoids in patients with severe COVID-19: a narrative review. Ann Transl Med 2021;9(15):1261. doi: 10.21037/atm21-1783 